\title{
Breast metastasis from a pulmonary adenocarcinoma: Case report and review of the literature
}

\author{
ALESSANDRO SANGUINETTI ${ }^{1}$, FRANCESCO PUMA ${ }^{2}$, ROBERTA LUCCHINI $^{1}$, STEFANO SANTOPRETE $^{2}$, \\ ROBERTO CIROCCHI ${ }^{3}$, ALESSIA CORSI $^{3}$, ROBERTA TRIOLA $^{1}$ and NICOLA AVENIA ${ }^{1}$ \\ ${ }^{1}$ Endocrine Surgical Unit, ${ }^{2}$ Thoracic Surgery Unit and ${ }^{3}$ General Surgery Unit, \\ University of Perugia, Umbria, Terni 06100, Italy
}

Received May 15, 2012; Accepted August 8, 2012

DOI: $10.3892 / \mathrm{ol} .2012 .995$

\begin{abstract}
Breast metastasis from extra-mammary malignancy is rare. An incidence of $0.4-1.3 \%$ has been reported in the literature. The primary malignancies most commonly metastasizing to the breast are leukemia, lymphoma and malignant melanoma. We present a case of metastasis to the breast from a pulmonary adenocarcinoma, diagnosed concomitantly with the primary tumor. A 43-year-old female presented with dyspnea and a dry cough of 3 weeks' duration. A subsequent chest radiograph revealed a massive pleural effusion. Additionally, on physical examination, a poorly defined mass was noted in the upper outer quadrant of the right breast. The patient underwent bronchoscopy, simple right mastectomy and medical thoracoscopy. Following cytology, histology and immunohistochemistry, primary lung adenocarcinoma with metastasis to the breast and parietal pleura was diagnosed. Histologically, both the primary and metastatic anatomic sites demonstrated a micropapillary component, which has recently been recognized as an important prognostic factor. Although the patient received chemotherapy, she succumbed to her condition within 8 months. Accurate differentiation of metastasis from primary carcinoma is very important as the treatment and prognosis of the two differ significantly.
\end{abstract}

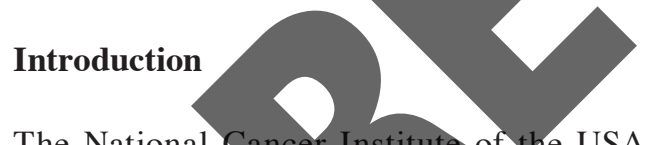

The National Cancer Institute of the USA estimates that, based on current rates, $12.7 \%$ of females born today will be diagnosed with breast cancer in their lifetime (1). Although primary breast cancer is the most common malignancy of adult females, metastatic involvement of the breast is rare, with a reported frequency of $0.4-1.3 \%$ in clinical series (2-5). Despite its rarity, metastatic breast disease is an significant diagnostic clinical problem, as its treatment differs greatly

Correspondence to: Dr Alessia Corsi, General Surgery Unit, University of Perugia, Umbria, Terni I-06100, Italy

E-mail: alessia.cor@libero.it

Key words: breast metastasis, lung cancer, micropapillary component, pulmonary adenocarcinoma from that of primary breast cancer. In 1907, Sitzentfrey was the first to publish a case of ovarian carcinoma metastatic to the breast (6). To date, a wide variety of malignancies have now been reported to metastasize to the breast and according to the literature, the most common primary tumors are melanomas and haematological malignancies (5-7). The lung is the most common cancer site in terms of incidence and mortality; however, there have only been a few published cases of pulmonary carcinomas metastasizing to the breast (8-12). Carcinomas with micropapillary components have been reported at several anatomical sites, including the breast, urinary bladder, ovary and major salivary glands (13). Micropapillary components are being increasingly recognized as prognostic predictors in lung adenocarcinomas and according to many studies, this may be a manifestation of aggressive behaviour $(14,15)$. We report the case of a patient with breast metastasis from a pulmonary adenocarcinoma characterized by a micropapillary pattern and diagnosed in conjunction with the primary tumor. Written informed consent was obtained from the patient for publication of this case report and accompanying images.

\section{Case report}

A 43-year-old, non-smoking housewife presented to the Emergency Department of St. Maria Hospital, Terni, Italy with dyspnea and a dry cough of 3 weeks' duration. A chest examination revealed reduced breath sounds and a percussive dullness in the left hemithorax. Physical examination revealed a painless, poorly defined mass, with associated skin redness, in the upper outer quadrant of the right breast. Palpable right axillary lymph nodes were also noted. A chest radiogram revealed a massive pleural effusion occupying the majority of the left hemithorax (Fig. 1A). Chest computed tomography (CT) (Fig. 1B) revealed the left lung to be atelectasic and compressed by a massive pleural effusion. The mediastinum and trachea were severely displaced to the right. A few lymph nodes were identified deep in the left axilla and a number of paratracheal lymph nodes were also observed. The clinical diagnosis was considered to be either a primary breast tumor with lung and pleural metastasis or two synchronous primary tumors. Mammography revealed a diffuse asymmetrical density in the sub alveolar region and the upper outer quadrant of the left breast (Fig. 1C). Additionally, skin thickening 
A
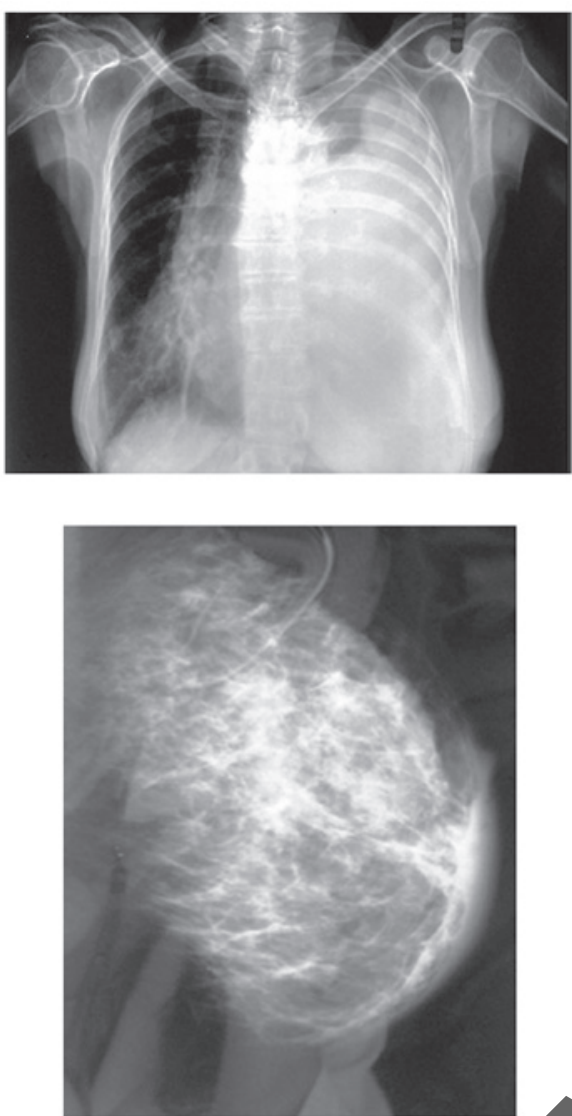

B

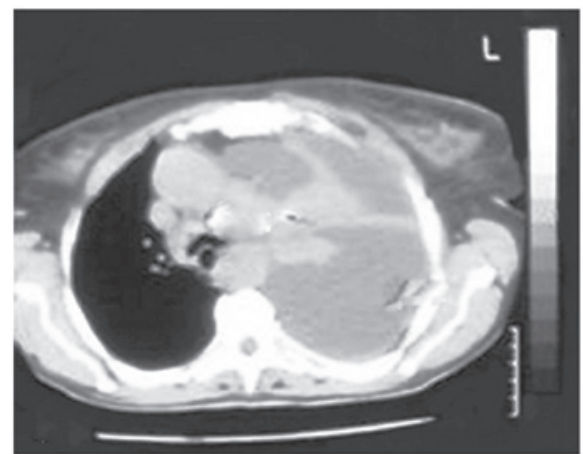

\section{C}

Figure 1. (A) Chest X-ray revealing a massive pleural effusion occupying the majority of the left hemithorax. (B) Chest CT revealing the left lung to be atelectasic and compressed by massive pleural effusion. (C) Mammography image revealing diffuse asymmetrical density in the subalveolar region and the upper outer quadrant of the left breast. (D) Chest CT revealing a $3.5 \times 4.5 \mathrm{~cm}$ peripheral lesion on the left upper lobe with relative abnormal contour and extension into the surrounding parenchyma.

was observed in the affected area/Calcifications were not observed. The differential diagnosis included inflammation, lymphoma and inflammatory breast carcinoma. The patient underwent bronchoscopy which revealed submucosal infiltration causing widening of the secondary carina and obstruction of the orifice of the lingula of approximately $70 \%$. Pleural effusion re-accumulated rapidly; therefore, in order to perform pleural drainage and chemical pleurodesis, medical thoracoscopy was carried out. During the procedure, biopsies were obtained from the parietal pleura. A new chest CT (Fig. 1D) followed and revealed a $3.5 \times 4.5 \mathrm{~cm}$ peripheral lesion on the left upper lobe, with relatively abnormal contours and extension into the surrounding parenchyma. The tumor was in contact with the splanchnic pleura and approached the parietal pleura. A right simple mastectomy was performed in order to remove the rapidly growing breast lesion. Our patient received 4 courses of bevacizumab, cisplatin and docetaxel with no clinical response. The patient succumbed to her condition 8 months after the diagnosis.

Cytological and immunocytochemical findings. All cytological specimens were stained by the Papanicolaou technique and evaluated by cytology. Following examination of the pleural effusion, bronchial washing and bronchial brush specimens, a diagnosis of adenocarcinoma was made. Immunocytochemistry performed on the smears prepared from the pleural effusion sample revealed the tumor cells to
D

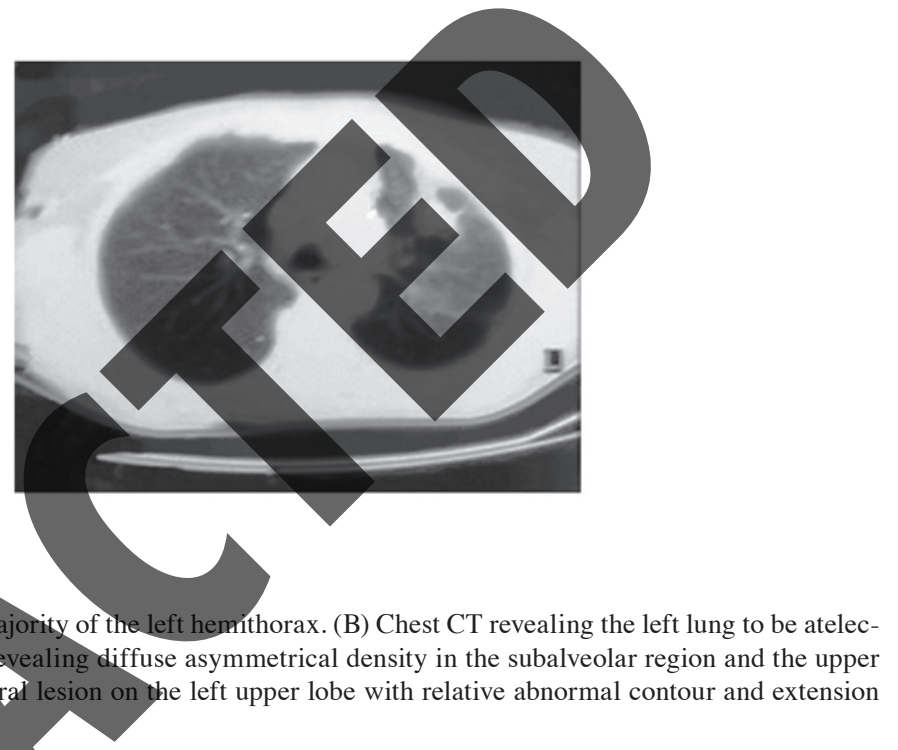

be strongly immunoreactive for thyroid transcription factor-1 (TTF-1) and monoclonal carcinoembryonic antigen (CEA). Tumor cells were negative for cytokeratin (CK) $5 / 6$, estrogen receptors (ER), cancer antigen (CA)-125 and thyroglobulin.

Histopathological and immunohistochemical findings. Hematoxylin and eosin (H\&E)-stained paraffin sections of the bronchoscopy biopsy revealed bronchial mucosal infiltration by a low differentiated adenocarcinoma. An extensive micropapillary component was identified (Fig. 2). This was observed as papillary structures with tufts that lacked a central fibrovascular core. In addition, occasional psammoma bodies were noted. Our differential diagnosis included primary lung adenocarcinoma, metastatic adenocarcinoma from the thyroid, breast or ovary and finally metastatic epithelioid (papillary) type-mesothelioma. The tumor cells demonstrated immunoreactivity for CD 15 (Leu-M1), TTF-1, surfactant protein A (SP-A) and monoclonal CEA. The neoplastic cells lacked expression of gross cystic disease fluid protein-15 (GCDFP-15), ER, mammaglobin, CK 5/6, calretinin, CA-125 and thyroglobulin. Based on the histology and the immunohistochemical staining patterns, a diagnosis of primary lung adenocarcinoma with a micropapillary component was made. H\&E-stained paraffin sections of the parietal pleura biopsies revealed diffuse infiltration by malignant epithelioid type cells, which demonstrated solid and micropapillary patterns. Additionally, numerous psammoma bodies were observed 


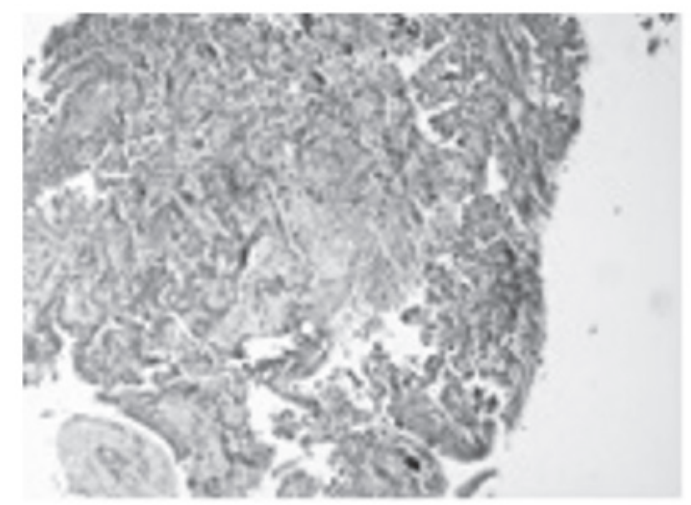

Figure 2. Hematoxylin and eosin (H\&E)-stained paraffin section of the bronchoscopy biopsy demonstrating bronchial mucosal infiltration by a low differentiated adenocarcinoma. An extensive micropapillary component was identified.

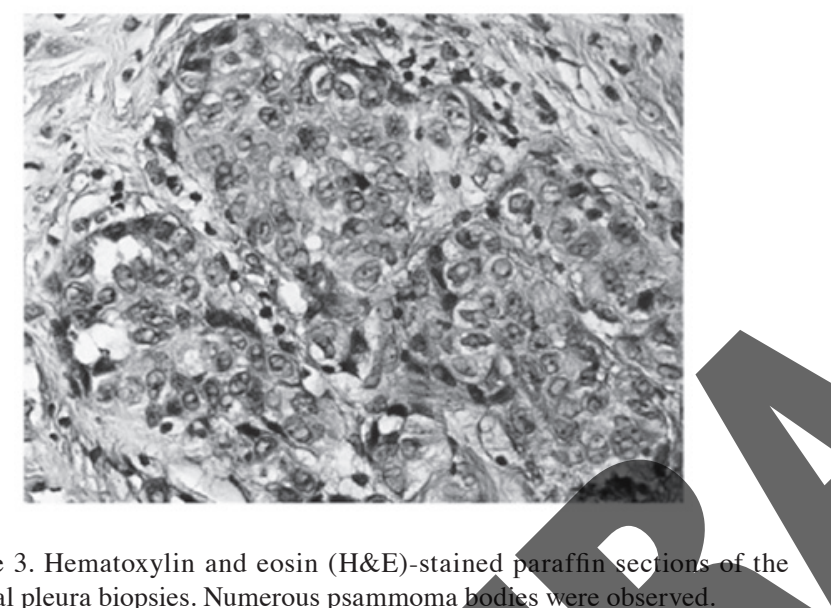

parietal pleura biopsies. Numerous psammoma bocic

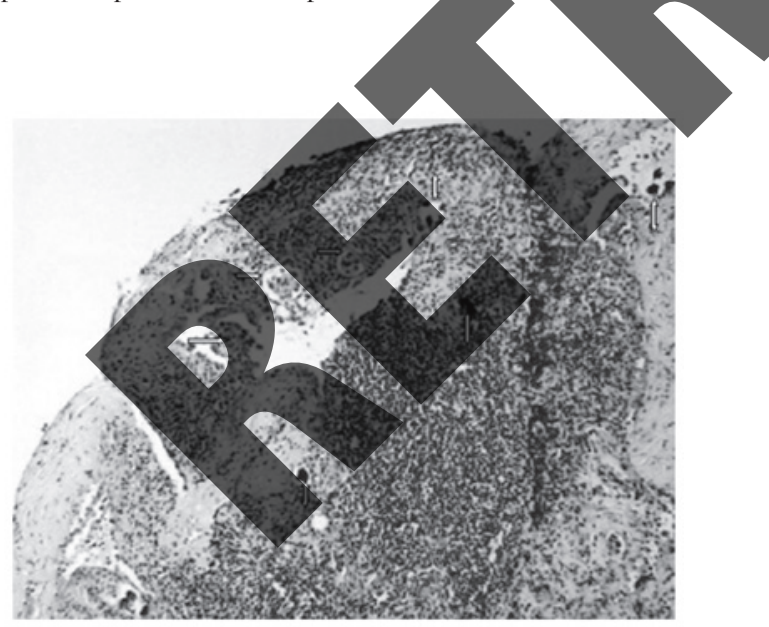

Figure 4. Breast biopsy specimen revealing dense fibrohyalinized stroma with atrophic terminal ductal lobular units. Within the stroma, sharply demarcated nodules of a high-grade adenocarcinoma with a solid and micropapillary pattern was demonstrated.

(Fig. 3). The tumor cells revealed the same immunoprofile as the lung biopsy. Finally, the breast biopsy specimen revealed dense fibro-hyalinized stroma with atrophic terminal ductal lobular units. Within the stroma, sharply demarcated nodules of a high-grade adenocarcinoma with a solid and micropapil- lary pattern were noted (Fig. 4). Lymphatic tumor emboli of micropapillary pattern adenocarcinoma with multiple psammoma bodies were also identified. The surrounding breast parenchyma demonstrated mild fibrocystic changes. Finally, no evidence of in situ carcinoma or elastosis was observed. Taking into account the diagnosis of the lung and pleura biopsies, our differential diagnosis included a second primary breast carcinoma and metastatic lung carcinoma. The tumor cells demonstrated immunoreactivity for TTF-1 and SP-A, and lacked expression of GCDFP-15, ER and mammaglobin.

\section{Discussion}

Worldwide, lung cancer is the most common cancer in terms of both incidence and mortality (1.6 million new cases per year and 1.378 million deaths) (8). Approximately one fifth of newly diagnosed lung adenocarcinomas present with distant metastases. The most common sites of metastasis are the brain, bone, liver and adrenal glands, in decreasing order. However, an autopsy series has demonstrated that non-small cell lung cancer (NSCLC) may spread to virtually any organ (16). Breast metastases from extra-mammary malignancies are rare, accounting for 0.4 to $1.3 \%$ of all breast malignancies (2-5). Approximately 700 cases have been reported in small series and case reports $(2-5,12,17,18)$. According to the international literature, the most common sources of primary tumors are hematological malignancies, malignant melanoma, lung tumors, renal cell carcinoma, ovarian tumors, thyroid carcinomas and small bowel carcinoids $(3,7,18)$. Williams et al published the largest series, which included 169 cases of metastases to the breast from extra mammary solid tumors, and reported that the most common histological type was malignant melanoma (7). A review of the literature (1990-2010) revealed approximately 30 NSCLC case reports or studies as part of a series of secondary breast tumors (4,5,9-12,19-27). Twelve of these cases were classified as adenocarcinomas $(5,9,12,19,21-23,25)$. Additionally, 53 cases of breast metastasis from lung tumors were presented; however, no detailed histological classification was provided (7,18,28-30). The majority of breast metastases present as palpable, rapidly growing, well-circumscribed and painless breast masses with predilection to the upper outer quadrant $(2,7,17,21,22)$. Unlike primary tumors, the vast majority of metastases do not demonstrate retraction of the skin or nipple, despite their superficial location $(5,22)$. However, in our patient, the lesion was poorly defined and skin redness was observed. Similar findings from other authors are rare $(7,17,25,30)$. Distinguishing a breast metastasis from a primary mammary adenocarcinoma, based on mammographic findings, may be extremely difficult due to the wide range of imaging manifestations of the metastatic lesions $(4,5,18)$. Thus, metastasis can mimic a primary malignancy or even a benign breast tumor $(4,5,18)$. The most commonly described mammographic presentation is usually single but may sometimes present as multiple well-circumscribed lesions with smooth margins $(3,18,30)$. Microcalcifications are very uncommon but have been reported in patients with metastatic serous ovarian papillary carcinoma $(18,29,30)$. In the present case, mammography revealed diffuse asymmetrical density and skin thickening. In similar cases, the differential 
diagnosis includes inflammation, lymphoma and inflammatory breast carcinoma. As cited in the literature, histological features that may aid in the recognition of secondary tumors include the absence of in situ carcinoma, which strongly supports a metastatic tumor, although this may not be present in all primary invasive carcinomas. Additionally, metastatic malignancies are often sharply circumscribed from the surrounding breast tissue. Furthermore, elastosis is common in primary tumors but rare in extra mammary malignancies $(2,4,5,12,19,21)$. Occasionally, metastases to the breast demonstrate features that lead pathologists to the correct diagnosis, such as the presence of pigmentation and intranuclear inclusions in malignant melanomas. Nevertheless, many extra mammary malignancies such as adenocarcinoma of the lung lack specific histological features. Carcinomas with a micropapillary component have been described in many organs including the breast, urinary bladder, ovary and salivary glands (13). In 2002, Amin et al were the first to report lung adenocarcinomas with micropapillary components (14). Histologically, the latter is characterized by small papillary tufts lying freely within alveolar spaces or encased within the thin walls of connective tissue. These small, cohesive nests lack fibrovascular connective tissue cores (14). In the present case, all biopsies examined demonstrated an extensive micropapillary component. Although psammoma bodies have not been observed in invasive micropapillary pattern carcinoma of the urinary bladder and salivary glands, they have occasionally been reported in cases of lung carcinoma with micropapillary morphology $(13,14,30,31)$ Multiple psammoma bodies were demonstrated in the tissue sections of the samples examined. To the best of our knowledge, this is the first report of breast metastasis from lung adenocarcinoma with a micropapillary pattern, diagnosed concomitantly with the primary tumor. The distinction between metastasis from lung adenocarcinoma, particularly with an extensive micropapillary pattern, and primary mammary adenocarcinomamay cause a significant diagnostic dilemma. The contribution of immunohistochemistry to the correct diagnosis is cracial. TTF-1 is expressed in $68-80 \%$ of lung adenocarcinomas, and with the exception of a single case published by Klingen et al (32), TTF-1 has not been reported to stain positively in breast adenocarcinoma (32-34). The sensitivity of SP-A is substantially less. It is expressed in approximately $45 \%$ of lung adenocarcinomas $(33,34)$. A negative expression of thyroglobulin excludes the diagnosis of papillary carcinoma of the thyroid, which stains positively for both markers. ERs are expressed in 80\% and GCDFP-15 in $45-53 \%$ of breast carcinomas $(33,35)$. Recent studies have revealed that ER expression in lung adenocarcinoma is low (7.6-14.1\%) by using the monoclonal antibodies 1D5 and 6 F11 $(33,36)$. Additionally, $5.2-15 \%$ of lung adenocarcinomas express GCDFP-15 (35,37). Finally, mammaglobin is expressed in $48-72.1 \%$ of mammary adenocarcinomas but stains negatively in pulmonary adenocarcinomas $(33,35,38)$. Consequently, a panel of markers must be used as no single antibody is $100 \%$ sensitive and false negative results do occur. In our case, all the tumor specimens (lung, pleura and breast) showed positive nuclear staining for TTF-1 and cytoplasmic staining for SP-A. The neoplastic cells lacked expression of GCDFP-15, ER and mammaglobin. Overall, metastasis to the breast has been associated with poor prognosis with the majority of patients succumbing to the disease within a year of diagnosis (7). Our patient survived for 6 months following the diagnosis of both the primary lung tumor and the breast metastasis.

Here, we reported a rare case of metastasis to the breast from an adenocarcinoma of the lung with an extensive micropapillary component. Metastatic disease to the breast, although rare, should be considered in the differential diagnosis of a primary mammary carcinoma as the treatment and prognosis differ significantly. Furthermore, the distinction between metastasis from lung adenocarcinoma, particularly with an extensive micropapillary pattern, and primary breast adenocarcinoma may cause a significant diagnostic dilemma. The contribution of immunohistochemistry to the correct diagnosis is essential.

\section{References}

1. National Cancer Institute: Probability of breast cancer in American women http://www.caneer.gov/cancertopics/factsheet/ detection/probability-breastcancer. Accessed March 9, 2012.

2. Hajdu SI and Urban JA: Cancers metastatic to the breast. Cancer 29: $1691-1696,1972$.

3. Vizcaino I, Torregrosa A, Higueras V, et al: Metastasis to the reast from extramammary malignancies: a report of four cases and a review of literature. Eur Radiol 11: 1659-1665, 2001 Georgiannos SN, ChinJ, Goode AW and Sheaff M: Secondary eoplasms of the breast: a survey of the 20th Century. Cancer 92: 2259-2266, 2001

5. Klingen TA, Klaasen $\mathrm{H}$, Aas $\mathrm{H}$, Chen $\mathrm{Y}$ and Akslen LA Secondary breast cancer: a 5-year population-based study with review of the literature. APMIS 117: 762-767, 2009.

6. Sitzenfrey A: Mammakarzinom zwei jahre nach abdominaler radikal operation wegen doppelseitigen carcinoma ovarii. Prag Med Wochenschr: 221-235, 1907 (In German).

Williams SA, Ehlers RA II, Hunt KK, et al: Metastases to the breast from nonbreast solid neoplasms: presentation and determinants of survival. Cancer 110: 731-737, 2007.

8. Ferlay J, Shin HR, Bray F, Forman D, Mathers C and Parkin DM: GLOBOCAN 2008 v1.2, Cancer Incidence and Mortality Worldwide: IARC CancerBase No. 10 [Internet]. Lyon, France: International Agency for Research on Cancer; 2010. http://globocan.iarc.fr. Accessed March 9, 2012.

9. Masmoudi A, Mathieu MC and Soria JC: Breast metastasis from lung adenocarcinoma: a case report. Anticancer Res 23: $1825-1826,2003$.

10. Ramar K, Pervez H, Potti A and Mehdi S: Breast metastasis from non-small-cell lung carcinoma. Med Oncol 20: 181-184, 2003.

11. Gomez-Caro A, Pinero A, Roca MJ, et al: Surgical treatment of solitary metastasis in the male breast from non-small cell lung cancer. Breast J 12: 366-367, 2006.

12. Lee AH: The histological diagnosis of metastases to the breast from extramammary malignancies. J Clin Pathol 60: 1333-1341, 2007.

13. Nassar H: Carcinomas with micropapillary morphology: clinical significance and current concepts. Adv Anat Pathol 11: 297-303, 2004.

14. Amin MB, Tamboli P, Merchant SH, et al: Micropapillary component in lung adenocarcinoma: a distinctive histologic feature with possible prognostic significance. Am J Surg Pathol 26: 358-364, 2002.

15. Maeda $\mathrm{R}$, Isowa $\mathrm{N}$, Onuma $\mathrm{H}$, et al: Lung adenocarcinomas with micropapillary components. Gen Thorac Cardiovasc Surg 57: 534-539, 2009.

16. Matthews MJ: Problems in morphology and behaviour of bronchopulmonary malignant disease. In: Lung Cancer: Natural History, Prognosis and Therapy. Isreal L and Chahanian P (eds). Academic Press, New York, pp.23-62, 1976.

17. Toombs BD and Kalisher L: Metastatic disease to the breast: clinical, pathologic, and radiographic features. AJR Am J Roentgenol 129: 673-676, 1977.

18. Noguera JJ, Martinez-Miravete P, Idoate F, et al: Metastases to the breast: a review of 33 cases. Australas Radiol 51: 133-138, 2007. 
19. Verger E, Conill C, Velasco M and Sole M: Metastasis in the male breast from a lung adenocarcinoma. Acta Oncol 31: 479, 1992.

20. Sadikot RT, Renwick DS, DaCosta P, Chalmers AG and Pearson SB: Breast metastasis from non-small cell lung cancer. South Med J 90: 1063-1064, 1997.

21. Lee SH, Park JM, Kook SH, Han BK and Moon WK: Metastatic tumors to the breast: mammographic and ultrasonographic findings. J Ultrasound Med 19: 257-262, 2000.

22. Yeh CN, Lin CH and Chen MF: Clinical and ultrasonographic characteristics of breast metastases from extramammary malignancies. Am Surg 70: 287-290, 2004.

23. Komorowski AL, Wysocki WM and Mitus J: Metastasis to the breast - a clinical challenge in outpatient. Acta Chir Belg 105: 59-61, 2005.

24. Ucar N, Kurt OK, Alpar S, Orsel O, Demirag F and Kurt B: Breast metastasis in a male patient with nonsmall cell lung carcinoma. South Med J 100: 850-851, 2007.

25. Fulciniti F, Losito S, Botti G, et al: Metastases to the breast: role of fine needle cytology samples. Our experience with nine cases in 2 years. Ann Oncol 19: 682-687, 2008.

26. Hsu W, Sheen-Chen SM, Wang JL, Huang CC and Ko SF: Squamous cell lung carcinoma metastatic to the breast. Anticancer Res 28: 1299-1301, 2008.

27. Wood B, Sterrett G, Frost F and Swarbrick N: Diagnosis of extramammary malignancy metastatic to the breast by fine needle biopsy. Pathology 40: 345-351, 2008.

28. Nielsen M, Andersen JA, Henriksen FW, et al: Metastases to the breast from extramammary carcinomas. Acta Pathol Microbiol Scand A 89: 251-256, 1981.

29. Muttarak M,Nimmonrat A and Chaiwun B: Metastatic carcinoma to the male and female breast. Australas Radiol 42: 16-19, 1998.
30. Lee SK, Kim WW, Kim SH, et al: Characteristics of metastasis in the breast from extramammary malignancies. J Surg Oncol 101: 137-140, 2010.

31. Kuroda N, Hamaguchi N, Takeuchi E, Ohara M, Hirouchi T and Mizuno K: Lung adenocarcinoma with a micropapillary pattern: a clinicopathological study of 25 cases. APMIS 114: 381-385, 2006.

32. Klingen TA, Chen Y, Gundersen MD, Aas H, Westre B and Sauer T: Thyroid transcription factor-1 positive primary breast cancer: a case report with review of the literature. Diagn Pathol 5: 37,2010

33. Yang $\mathrm{M}$ and Nonaka D: A study of immunohistochemical differential expression in pulmonary and mammary carcinomas. Mod Pathol 23: 654-661, 2010

34. Zamecnik J and Kodet R: Value of thyroid transcription factor-1 and surfactant apoprotein $\mathrm{A}$ in the differential diagnosis of pulmonary carcinomas: a study of 109 cases. Virchows Arch 440: 353-361, 2002.

35. Takeda Y, Tsuta K, Shibuki Y, et al: Analysis of expression patterns of breast cancer-specific markers (mammaglobin and gross cystic disease fluid protein 15) in lung and pleural tumors. Arch Pathol Lab Med 132: 239-243, 2008.

36. Gomez-Fernandez C Mejias A, Walker G and Nadji M: Immunohistochemical expression of estrogen receptor in adenocarcinomas of the lung: the antibody factor. Appl Immunohistochem Mol Morphol 18: 137-141, 2010.

37. Striebel JM, Dacic S and Yousem SA: Gross cystic disease fluid protein-(GCDFP-15): expression in primary lung adenocarcinoma. Am J Surg Rathol 32: 426-432, 2008

38. Bhargava R, Beriwal S and Dabbs DJ: Mammaglobin vs GCDFP-15: an immunohistologic validation survey for sensitivity and specificity. Am J Clin Pathol 127: 103-113, 2007. 